\title{
TV/Series
}

$14 \mid 2018$

Posthumains en séries

\section{Posthumain et réception, de The Tempest à Westworld}

Delphine Lemonnier-Texier

\section{OpenEdition}

1 Journals

Édition électronique

URL : http://journals.openedition.org/tvseries/2786

DOI : 10.4000/tvseries.2786

ISSN : 2266-0909

Éditeur

GRIC - Groupe de recherche Identités et Cultures

Référence électronique

Delphine Lemonnier-Texier, « Posthumain et réception, de The Tempest à Westworld », TV/Series [En ligne], 14 | 2018, mis en ligne le 31 décembre 2018, consulté le 30 avril 2019. URL : http:// journals.openedition.org/tvseries/2786; DOI : 10.4000/tvseries.2786

Ce document a été généré automatiquement le 30 avril 2019.

\section{(c) $(1) \Theta \Theta$}

TV/Series est mis à disposition selon les termes de la licence Creative Commons Attribution - Pas d'Utilisation Commerciale - Pas de Modification 4.0 International. 


\title{
Posthumain et réception, de The Tempest à Westworld
}

\author{
Delphine Lemonnier-Texier
}

1 Comme le souligne Paul Booth, le personnage est au cœur du récit télévisé ${ }^{1}$. Il est l'instrument qui permet l'identification, définie comme la capacité à produire sur le spectateur un effet qui modifie sa posture de réception: «Identification doesn't have to be about being like or liking a character, but rather about having that character emotionally move an audience ${ }^{2}$." Dans la fiction télévisuelle, le personnage est un substitut du spectateur ( a surrogate for the viewer ${ }^{3} »$ ). Par le processus d'identification, le spectateur se trouve comme en immersion dans l'univers fictionnel, dont il fait l'expérience en même temps que le personnage: "[...] there is a form of identity augmentation between character and viewer. The knowledge that the characters gain augments the audience member's knowledge ${ }^{4}$ ». C'est ce processus de compagnonnage avec le personnage qui définit la posture de la spectatrice ${ }^{5}$ implicite inscrit dans le texte du récit télévisuel.

2 Lorsque l'univers fictionnel est posthumain, c'est-à-dire à partir des années $1950^{6}$, la fiction met en scène la question du statut ontologique du robot et la nature de la relation qui s'établit entre lui et le personnage humain, en jouant sur des effets de "vallée de l'étrange " entre les différents niveaux du récit télévisuel. Phénomène postulé par Masahiro Mori concernant l'interaction entre humains et robots dans le monde réel, la "vallée de l'étrange » qualifie un effet de répulsion suscité par un artefact à l'apparence humaine très convaincante :

\footnotetext{
Néanmoins, ce type de main prosthétique est trop réaliste et au moment où nous réalisons qu'elle est artificielle, un sentiment d'étrangeté s'installe en nous. Lorsque nous serrons cette main, nous sommes surpris par l'absence de tissus mous et par sa froideur. Le sentiment de familiarité disparaît pour être remplacé par un sentiment d'inquiétante étrangeté ${ }^{7}$.
}

Il ressort de cet exemple expliqué par Mori deux éléments marquants : en premier lieu, l'effet d'étrangeté est d'autant plus intense que l'illusion initiale semblait parfaite; en second lieu, le sentiment de "vallée de l'étrange » est suscité par le hiatus entre deux 
étapes distinctes de perception sensorielle d'un même objet (dans le cas présent, la vue et le toucher). Dans la fiction filmique ou télévisuelle, où il n'est possible de solliciter que la vue et l'ouie du spectateur, on pourra parler d'effets de " vallée de l'étrange » en lien avec la question de la vraisemblance et de l'engagement affectif du spectateur, puisque, comme le souligne Anaïs Goudmand : "L'impression de vraisemblance qui se dégage du récit fonctionne comme un opérateur d'immersion qui engage affectivement le récepteur ${ }^{8}$ . » Des effets de "vallée de l'étrange » se produisent donc à des moments de rupture qui rendent visibles les rouages de l'illusion, au sens figuré comme au sens propre : l'artefact robotique étant manifeste à l'image, c'est tout le processus de réception qui se trouve décalé par le questionnement du vraisemblable qui en découle. La rupture de l'illusion dans la fiction interroge le spectateur sur ce qui l'a amené à choisir d'y croire. Tout ce qui précède ce moment de rupture est susceptible d'être revisité et réinterprété, et un miroir est tendu au spectateur en tant qu'acteur du processus de réception. Au cœur du dispositif se trouvent donc deux éléments incontournables : la question du vraisemblable dans la fiction, et le catalyseur que constitue l'empathie.

4 La fiction télévisuelle s'empare très tôt de cet effet de "vallée de l'étrange ", par exemple avec « The Lonely ", l'épisode 7 de la saison 1 de The Twilight Zone ${ }^{9}$. Corry, condamné par la justice pour un meurtre qu'il n'a pas commis à passer plusieurs années sur une planète déserte, sait parfaitement qu'Alicia, la gynoïde qu'on lui a accordée pour adoucir quelque peu sa peine, est un robot. Il commence par la rejeter violemment, n'étant que trop conscient du leurre d'humanité que la machine constitue dans l'immensité de sa solitude. Cependant, touché par le fait qu'elle éprouve des sentiments et pleure lorsqu'il la rejette, il finit par la considérer comme une compagne humaine, dont elle présente tous les comportements et caractéristiques. Tout comme Corry, la spectatrice est confrontée aux larmes d'Alicia, et l'empathie que ressent le personnage invite la spectatrice à accepter également la vraisemblance de la souffrance de cette dernière (cf Fig. 1).

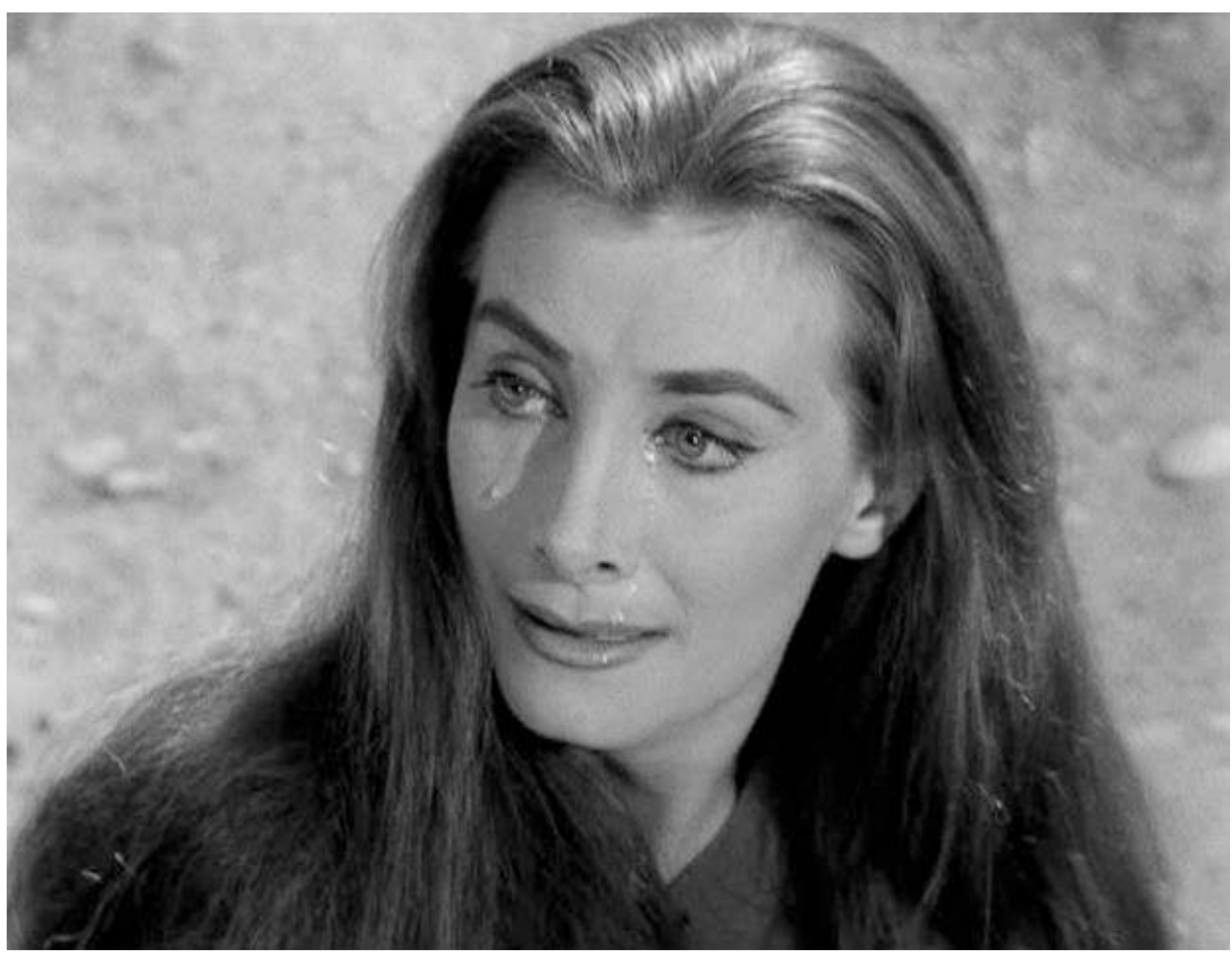

Fig. 1 : Les larmes d'Alicia 
Lorsque les hommes venus rechercher Corry après la suspension de sa peine le retrouvent, il refuse de laisser Alicia et exige de la ramener sur Terre, alors que la fusée ne le permet pas faute de place. Pour ramener Corry à la raison, un coup de feu est tiré sur Alicia. Son visage déchiqueté devenu trou béant laisse alors voir les circuits intégrés et les fils qui constituent l'architecture interne de la machine, choc dont Corry peine à se remettre, tant l'humanisation d'Alicia la rendait jusqu'alors en tous points semblable à une femme " parfaite ». Le cadrage identique met en parallèle le visage en larmes d'Alicia et la machine mise à nu (cf. Fig 2), si bien que l'empathie et le choc de Corry invitent, de nouveau, à des ressentis similaires chez le spectateur.

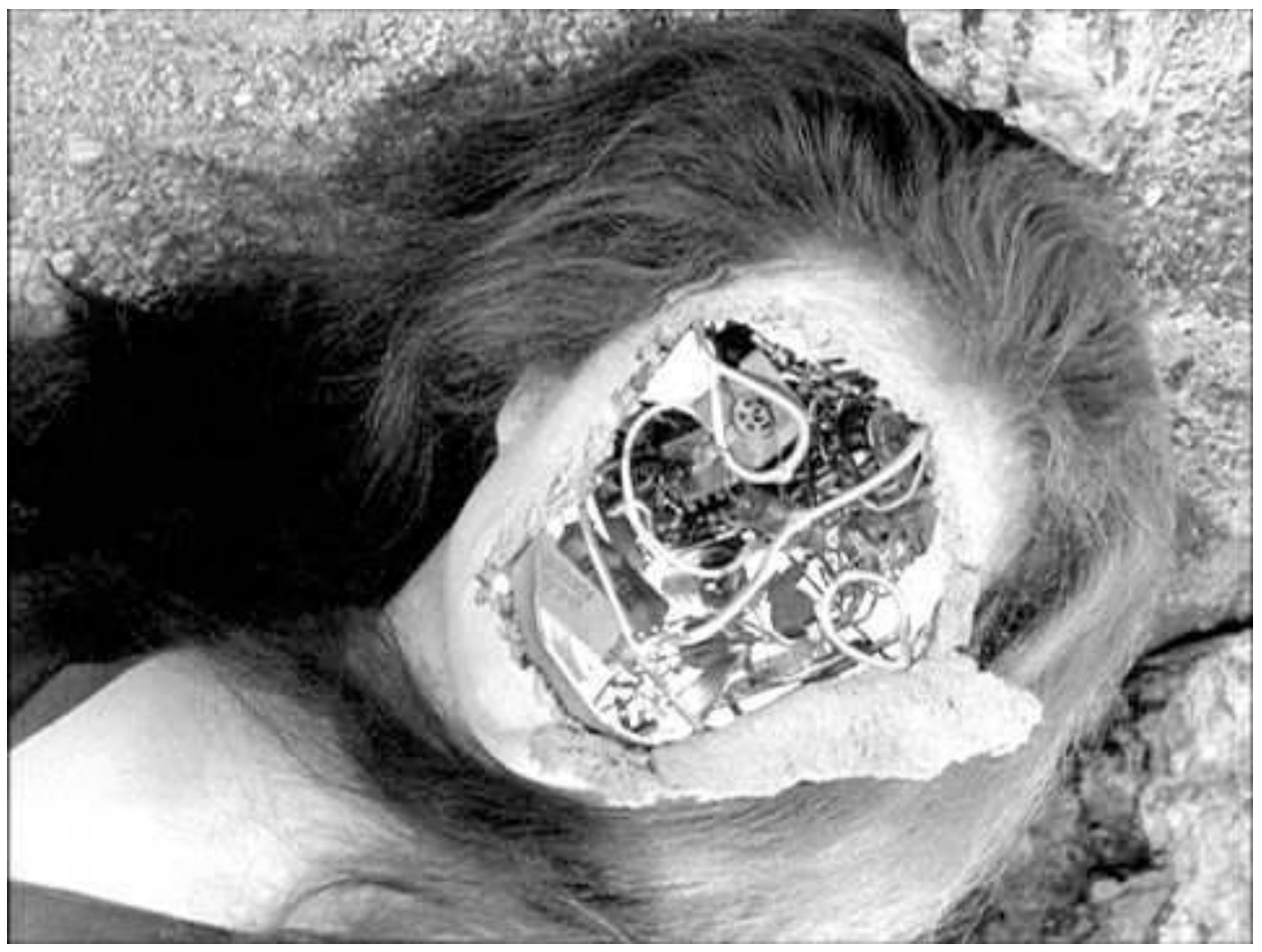

Fig. 2 : La machine mise à nu

Le moment de la «vallée de l'étrange» est donc un outil narratif qui souligne l'importance de l'empathie du spectateur, dont celle de Corry est le miroir, en dépit de la parfaite connaissance que tous deux ont de la véritable nature d'Alicia dès son apparition dans l'épisode. Stockée dans une boîte, elle est présentée à Corry comme une machine similaire à la voiture que ce dernier a passé de longs mois à remettre en état. Dès lors qu'elle s'anime et ressent de la souffrance, l'empathie déclenche donc un processus de suspension volontaire de l'incrédulité, un effet de "je sais bien mais quand même ${ }^{10}$ ", auquel le moment de "vallée de l'étrange » viendra brutalement mettre fin. Cet épisode fonctionne donc sur un double déplacement de la posture de la spectatrice : lorsque le robot s'anime et souffre, l'impression de vraisemblance immerge la spectatrice dans le récit et l'engage affectivement au même titre que le personnage de Corry, par le biais de l'empathie, en dépit de sa parfaite connaissance de la véritable nature d'Alicia. Cette immersion dans l'univers fictionnel est évidemment d'autant plus aisée que le personnage de gynoïde est joué par un être humain. Et à l'inverse, le brutal rappel de cette nature machinique au moment du plan sur le visage déchiqueté brise le cœur de Corry (qui devient alors objet potentiel d'empathie pour les spectateurs) et re-décale la position des spectateurs vers une dimension réflexive. 
7 Comme The Tempest, qui est aussi le premier intertexte shakespearien cité par le personnage de Peter Abernathy dans l'épisode 1 lorsqu'il dit à Dolores (qui est censément sa fille) "You should go. Leave. Don't you see ? Hell is empty and all the devils are here ", la série Westworld ${ }^{11}$ place dans sa saison 1 les personnages des hôtes (les robots du parc d'attraction) en situation d'ironie dramatique tout en multipliant leurs moments de souffrance extrême. Contrairement aux techniciens de Delos et aux spectateurs de la série, ils ignorent leur véritable nature et se croient humains. La série use du procédé de "vallée de l'étrange » de manière classique, mais aussi de manière inattendue, en confrontant les hôtes à leur caractère artificiel, ce qui génère chez eux un refus accompagné de révulsion, afin de questionner, comme le fait la pièce de Shakespeare, le spectateur sur sa propre (in)humanité.

\section{Empathie et ironie dramatique dans The Tempest}

8 Il peut sembler quelque peu paradoxal de parler de posthumain dans The Tempest au premier abord. Pourtant, cette pièce atypique présente plusieurs caractéristiques qui s'apparentent à la notion. Comme l'a démontré Kevin LaGrandeur, dans le Dr. Faustus de Christopher Marlowe et dans The Tempest de William Shakespeare, on trouve des préfigurations du sujet posthumain :

The intelligent servant network created by Prospero in The Tempest and Marlowe's protagonist in Dr. Faustus provide their respective makers with not only an enhancement but also a distribution of their agency. They represent proxies for their makers and so can be seen not just as prostheses but as distributed, networked versions of their makers' selves-in a sense as early modern predecessors of the contemporary posthuman subject ${ }^{12}$.

9 Ariel est ainsi l'interface qui gouverne une petite armée d'esprits au service de Prospero, auquel il est lui-même assujetti. Grâce à ce dispositif, Prospero contrôle tout ce qui se produit sur son île et agit à distance sur tous les êtres vivants qui la peuplent, même s'il demeure à tout moment possible que l'une de ses créatures décide de se rebeller contre lui :

But books are not the only source of Prospero's power to create and control his intelligent servant system. Nor are they sufficient because the system's power is so great that the master-slave relationship is unstable between it and the magician. This plot point anticipates posthuman concerns that appear in today's fictional and factual literature ${ }^{13}$.

Dans The Tempest, « Hell is empty and all the devils are here » est déjà une citation. C'est Ferdinand qui aurait prononcé ces paroles, selon le récit que fait Ariel à Prospero du déroulement de la tempête ${ }^{14}$. Ce récit s'inscrit en complément, et non en résumé, de la première scène de la pièce où le spectateur a assisté à cette tempête et au naufrage qu'elle a provoqué. Or cette scène, dont Miranda (incarnation du regard empathique) a été spectatrice, et dont elle a cru, comme les spectateurs de la pièce, qu'elle signifiait la mort de tous ceux qui se trouvaient sur le bateau, était en réalité une illusion créée par la magie de Prospero. Shakespeare construit donc une scène d'exposition tronquée qui souligne le mode habituel de la réception du spectacle de théâtre pour le mettre à distance de manière réflexive : ce que montre l'acte 1 scène 1 est en réalité un piège qui trompe aussi bien les personnages qui le vivent que Miranda et, avec elle, tous les 
spectateurs de la pièce. Cette citation de Ferdinand illustre donc le choix fait par Shakespeare dans cette pièce, celui de l'ironie dramatique. À partir de l'acte 1 scène 2, et à l'exception de Prospero, tous les êtres humains sur l'île sont en situation d'ironie dramatique, outil majeur de la fiction théâtrale comme le souligne Jonathan Hart :

Prospero's magic is connected with that most powerful of theatrical toolsdramatic irony, which creates a bond between the playwright and the audience, a connection that calls for superior knowledge of the characters. One variant of dramatic irony is when the dramatist and the audience share superior knowledge with one or more characters and together they are aware of the ignorance of other characters ${ }^{15}$.

11 Sur l'île de Prospero, tout est artificiel, tout est illusion: une simple injonction verbale met fin à l'effusion d'émotions de Miranda pour la plonger dans un profond sommeil :

Here cease more questions:

Thou art inclined to sleep; 'tis a good dulness,

And give it way: I know thou canst not choose. (1.2.185-187)

12 Le récit d'Ariel révèle que les morts sont bien vivants, et même régénérés dans leur apparence :

ARIEL. Not a hair perish'd;

On their sustaining garments not a blemish,

But fresher than before (1.2.218-220)

La structure de la pièce, avec le dédoublement de la scène d'exposition (la première scène construite comme un dénouement tragique; la seconde comme la déconstruction de la première et la révélation des rouages de l'illusion), révèle un univers fictionnel avec ses lois propres, radicalement différentes du monde réel des spectateurs, un univers où l'on peut revenir d'entre les morts, faire taire un afflux d'émotions d'une simple injonction et faire revenir un personnage très malmené physiquement à une apparence parfaitement intacte. Du point de vue de la réception, c'est un univers marqué par l'ironie dramatique, puisque les personnages croient à la véracité des événements qu'ils vivent et des chagrins qui les affectent, cependant que le spectateur, lui, est averti du caractère illusoire de l'ensemble. Mais c'est aussi un univers marqué par l'empathie, et par l'existence de points de vue narratifs fortement divergents du point de vue dominant, celui de Prospero, caractéristique qui rapproche la pièce de l'Enéide de Virgile, comme l'a montré Leah Whittington ${ }^{16}$.

14 C'est sur un schéma global du même type qu'est construite la saison 1 de Westworld: les hôtes se croient humains et pensent vivre d'authentiques événements et de vraies vies. Et la spectatrice sait que ce sont en réalité Ford, Bernard et, dans une moindre mesure, Sizemore qui contrôlent l'intégralité de leurs faits et gestes, qui sont en outre répétés et répétables quasiment à l'infini.

15 L'île de Prospero, comme le parc de Delos ${ }^{17}$, est un espace clos compartimenté en plusieurs sections, où sont positionnés des personnages différents, chacun inscrit dans un fil narratif distinct. La tentative de révolte contre le tout-puissant Prospero émerge chez les créatures qu'il a réduites en esclavage (Caliban, Ariel) aussi bien que chez sa propre fille. L'intrigue démontre la toute-puissance de Prospero et sa capacité à parvenir à ses fins. Pour autant, la pièce se termine, contre toute attente, par un discours de renonciation, un monologue adressé directement au spectateur. Prospero y tire sa révérence en remettant entre les mains des spectateurs la responsabilité du choix d'un dénouement laissé inachevé. L'épisode final de la saison 1 de Westworld est construit selon 
la même logique et théâtralise fortement la présentation par Ford de son ultime récit, en faisant figurer des spectateurs, les membres de la direction de Delos. Ce dispositif est déployé aussi bien sur la plage, avec la scène finale du voyage de Teddy et de Dolores, que dans le village d'Escalante pour le monologue final de Ford. Pour la scène sur la plage, les artifices du récit que Ford y déroule sont fortement soulignés: la lune au-dessus de l'horizon n'est finalement qu'un projecteur de cinéma, les deux personnages sont des robots qu'il faut nettoyer et réparer, et des assistants viennent déconstruire l'ensemble aussitôt que Ford a stoppé l'action en figeant Dolores et Teddy, tout comme les assistants de Prospero démontent les éléments du Masque dans The Tempest après que Prospero leur en a intimé l'ordre («Well done! Avoid; no more! », 4.1.142). La seconde partie du discours de Ford rappelle aux membres de Delos qu'ils sont avec lui « in a prison of our own sins ${ }^{18}$ ", tout comme, dans The Tempest, la scène du banquet s'achève au moment où Ariel rappelle aux spectateurs sur scène, Antonio, Sebastian et Gonzalo, qu'ils sont « three men of sin» (3.3.53). Dans l'épisode 1, après avoir été interrogé par Ford, Peter Abernathy s'était soudain jeté sur lui en lui disant "You are in a prison of your own sins ». La phrase de Ford dans l'épisode 10 constitue donc un intratexte qui fait le lien entre l'épisode pilote et le final de la saison 1 pour souligner, par l'usage de la première personne du pluriel, le changement de statut de la citation, devenue inclusive : Ford se range du côté de ses spectateurs, s'incluant dans un «nous » qui renvoie à l'ensemble du genre humain, comme s'il avait implicitement accepté la véracité de l'accusation d'Abernathy.

Un moment de rupture similaire se produit dans The Tempest à l'acte 5 , scène 1 . Toutpuissant et tyrannique, Prospero semble prendre plaisir à faire excessivement souffrir les autres. Il se retrouve confronté simultanément au rappel de la non-humanité d'Ariel, équivalent verbal du moment de « vallée de l'étrange » du récit filmique posthumain, et à une leçon fondamentale d'empathie :

ARIEL. Him that you termed, sir, 'The good old lord Gonzalo;'

His tears run down his beard, like winter's drops

From eaves of reeds. Your charm so strongly works 'em

That if you now beheld them, your affections

Would become tender.

PROSPERO. Dost thou think so, spirit?

ARIEL. Mine would, sir, were I human.

PROSPERO. And mine shall.

Hast thou, which art but air, a touch, a feeling

of their afflictions, and shall not myself,

One of their kind, that relish all as sharply

Passion as they, be kindlier moved than thou art? (5.1.15-24 $\left.{ }^{19}\right)$

Cette définition de l'humain comme fondamentalement doué d'empathie inflige à Prospero une véritable leçon sur sa propre inhumanité et provoque son retournement. Honteux de son insensibilité face à l'empathie d'un être qui n'en est théoriquement pas capable, il change radicalement de posture pour à la fois renoncer à ses pouvoirs magiques et reconnaître ses torts dans le monologue final où il demande à être lui-même pardonné pour ses péchés ( « As you from crimes would pardon'd be / Let your indulgence set me free $\left.{ }^{20} »\right)$. Il se retrouve donc exactement dans la position de ceux qui étaient précédemment ses victimes, les hommes qui avaient causé sa déchéance, réduits par le pouvoir de sa magie sur l'île à se repentir de l'avoir trahi. 


\section{« Vallée de l'étrange » et empathie dans Westworld}

18 C'est avec une relative parcimonie que la première saison de la série Westworld fait usage des classiques images de corps artificiels et de la confrontation avec la fascinationrépulsion exercée par la mise à nu de leur mécanisme. On peut ainsi penser, outre les images de fabrication des squelettes synthétiques, à Old Bill et à ses gestes saccadés (S01E01 et S01E05), à Abernathy et au shérif avec leurs dysfonctionnements physiques et verbaux (S01E01), ou encore au petit Robert, réplique de Ford lorsqu'il était enfant. Lorsque Ford lui ordonne "Turn the other cheek» (S01E06), le garçonnet révèle la machine de première génération cachée sous son visage d'enfant (cf. Fig. 3).

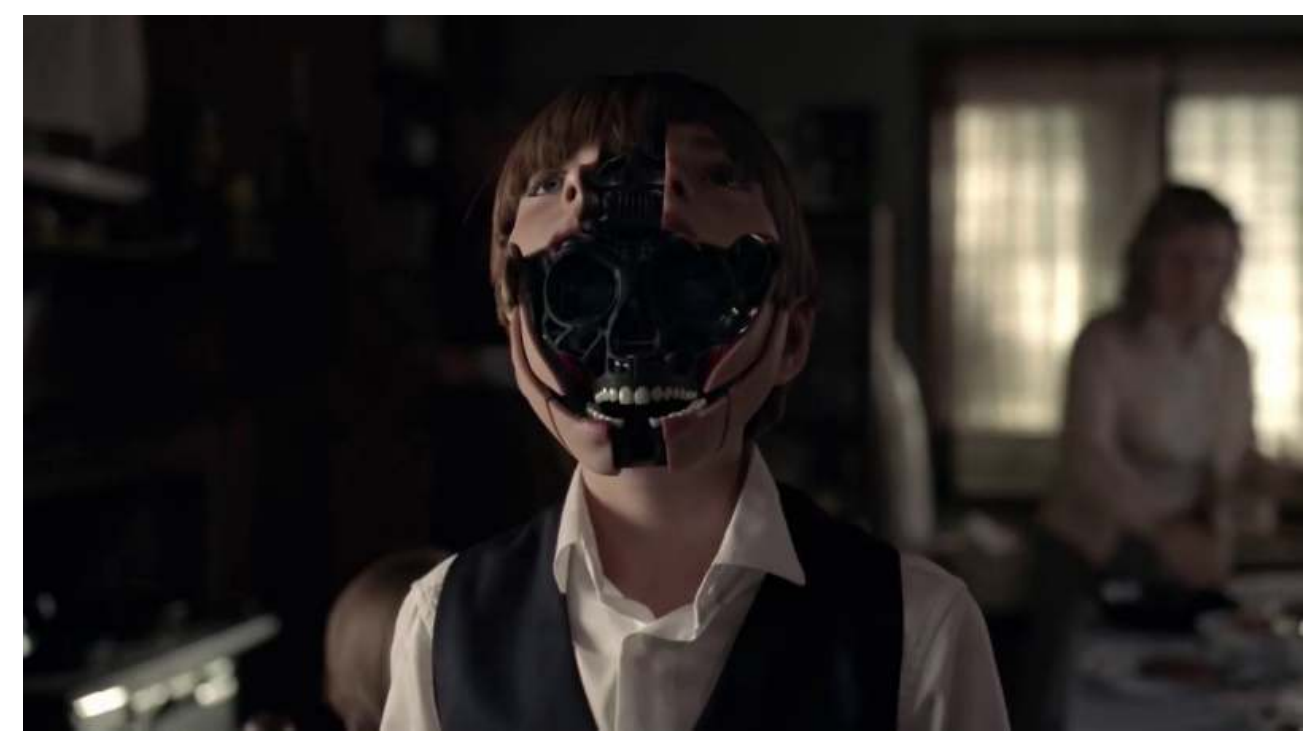

Fig. 3 : La machine sous le visage de l'enfant

19 Westworld déploie surtout une utilisation plus radicale des effets de "vallée de l'étrange » en situant ceux-ci du point de vue des hôtes qui découvrent l'impensable réalité de leur caractère artificiel.

De manière remarquable, Shakespeare demeure dans la série le point de référence explicite pour définir la notion d'humain et de posthumain, comme le souligne Ford luimême dans une conversation avec Teddy dans l'épisode 3 de la saison 1 où il cite Julius Caesar:

A coward dies a thousand deaths. The valiant taste of death but once. Of course, Shakespeare never met a man quite like you, Teddy. You've died at least a thousand times. And yet, it doesn't dull your courage. Tell me, is that all you aspire to, Teddy?

21 Après avoir été confrontée à sa propre artificialité dans l'épisode 7, Maeve comprend la supériorité qui est la sienne. Elle reprend ce même argument face à Sylvester : "You think I'm scared of death? I've done it a million times. I'm fucking great at it. How many times have you died?»

La série déploie tout un éventail d'images de la fabrication et de la maintenance des robots du parc, comme le fait le film-source de Michael Crichton en 1973, mais avec une spécificité marquée, qui rapproche le plus possible les hôtes d'une apparence humaine. Le générique est ainsi composé d'images de squelettes synthétiques animés sur le mode d'une étrange danse macabre ou d'un memento mori posthumains (la cavalière et son 
cheval, les mains sur le clavier du piano). L'épisode pilote montre plus en détail la manufacture des hôtes, avec un squelette dans la posture emblématique de l'homme de Vitruve que l'on plonge dans un bain de liquide blanc sous le regard contemplatif de Ford. À ces éléments humanistes clairement référencés, il faut ajouter le traitement visuel des blessures infligées aux hôtes par les invités du parc, avec des gros plans sur des chairs déchiquetées et plaies sanguinolentes, c'est-à-dire semblables à des blessures infligées à des corps humains. Le cheminement de l'épisode pilote part donc du simulacre d'humanité des robots (leur architecture osseuse et musculaire) pour déboucher sur leur très forte humanisation, et à cet égard, la comparaison des séquences initiale et finale est éloquente.

Dans la séquence initiale, on découvre Dolores immobile et plongée dans la pénombre. Une voix off ordonne "bring her back online", mais ce sont les néons environnants qui s'allument, cependant qu'à l'image Dolores demeure figée. Sa voix, également off, commence à répondre aux questions d'Arnold, et leur dialogue est en complet décalage avec l'image où elle apparaît rigoureusement immobile. Une mouche court sur son front, et la caméra zoome rapidement, accompagnée d'une musique qui souligne le gros plan, pour culminer au moment où la mouche se promène sur sa pupille, figée comme l'œil de porcelaine d'une poupée. En même temps que cette séquence de construction du personnage gynoïde efface l'humanité de l'actrice jouant le rôle, la suite souligne le degré extrême d'ironie dramatique qui caractérise Dolores, convaincue de la bonté naturelle des nouveaux-venus au sein de son monde, ceux qu'elle appelle les «newcomers». En effet, tandis que les images montrent comment ces derniers abusent d'elle et des autres hôtes avant de les massacrer, sa voix affirme l'amour universel qu'ils inspirent aux robots « we all love the newcomers ». Dans la séquence finale de l'épisode, Dolores est revenue dans son monde après avoir été interrogée par les techniciens suite au dysfonctionnement majeur de son père, Peter Abernathy. Désormais capable de mentir aux techniciens, à qui elle a assuré qu'elle ne ferait jamais de mal à une créature vivante, elle écrase immédiatement la mouche qui se pose sur son cou. La claque interrompt brutalement la musique harmonieuse qui accompagne le mantra de son discours idéaliste (en voix off) sur la beauté de son monde, et son sourire s'efface alors qu'elle semble jeter pour la première fois un regard libre sur le monde qui l'environne, avant un fondu au noir qui précède le générique de fin. Démenti flagrant apporté aux certitudes des techniciens quant à l'innocuité des robots (un peu plus tôt dans ce même épisode, Bernard affirmait à Theresa Cullen, au sujet du shérif : " he literally couldn't hurt a fly »), cette séquence de clôture renverse donc complètement la situation d'ironie dramatique initiale et rend possible une réception fondée sur le compagnonnage des personnages des hôtes avec les spectateurs dans les épisodes suivants. Avec la complicité des spectateurs, ce sont désormais les humains employés par Delos qui se trouvent en situation d'ironie dramatique par rapport à Dolores.

Par ailleurs, il est manifeste que deux temporalités du posthumain sont présentes dans l'épisode pilote, celle du passé du parc, et celle de son présent. Le passé du parc est représenté par le robot aux gestes saccadés et aux paroles répétitives, ancien modèle remisé au sous-sol de la Mesa (au niveau -B83), old Bill. Vieille légende de l'Ouest américain comme le souligne son nom, il est avant tout âgé, et seul personnage historique du parc (Wild Bill Hickok). Ses mouvements saccadés, répétitifs, et sa diction tout aussi peu variée l'ancrent dans les clichés des premières heures de la robotique, et son corps porte tous les stigmates du grand âge humain. Ce dernier élément constitue une touche 
supplémentaire qui humanise encore davantage les robots. À l'opposé de Bill se trouve la peau fraîche et lisse de Clementine, une gynoïde si parfaite, aux gestes si précis et harmonieux que même la technicienne chargée de sa maintenance, Elsie Hughes, se laisse emporter par une pulsion et l'embrasse sur la bouche, illustrant la véracité du constat de Bernard quelques instants auparavant : «It's the tiny things that make them seem real, that make the guests fall in love with them. »

Ces séquences montrent le cheminement de l'épisode pilote. Après lui avoir montré le fonctionnement de la fabrique des robots depuis les balbutiements des appareils de première génération, l'épisode emmène le spectateur vers une suspension volontaire de l'incrédulité face à des hôtes qui réunissent les critères de la parfaite illusion mimétique et sont, au même titre que l'homme en noir - fidèle visiteur du parc depuis trente ans, qui semble prendre un plaisir particulier à abuser des robots et à les abattre - des personnages centraux du récit.

Il s'agit là d'une évolution majeure de la série par rapport au film de 1973, où le spectateur suivait depuis la séquence d'ouverture du film le parcours des humains qui visitaient le parc. Dans le pilote, c'est Teddy que l'on suit, plusieurs fois de suite, à son arrivée à Sweetwater. C'est aussi Dolores que l'on voit se réveiller le matin dans son lit, allongée sur un oreiller blanc tandis que, dans le film de Crichton, ce même plan du personnage qui se réveille dans son lit était utilisé exclusivement pour les visiteurs du parc.

Cette évolution explique sans doute que les effets de "vallée de l'étrange » soient déployés dans la saison 1 sur deux parcours remarquables de personnages, l'un humain, et l'autre posthumain.

Le premier concerne le jeune William, qui se retrouve devant Dolores comme Elsie Hughes face à Clementine, en dépit des mises en garde réitérées de Logan («She’s a goddamn doll », S01E05). Depuis son arrivée dans le parc, William refuse d'écouter Logan et de traiter les hôtes comme des machines insensibles. Au contraire, il leur prête attention, écoute ce qu'ils lui racontent et analyse leurs réactions. Face à Dolores, non seulement il tombe amoureux mais il décèle chez elle des signes d'une différence par rapport aux autres robots, d'un début de conscience. William et Logan incarnent donc pour les spectateurs deux postures opposées de réception. Trahi par William au profit de Dolores, Logan décide alors de passer à l'acte pour contraindre William à regarder la vérité en face. Joignant le geste à la parole («Perhaps a more visceral demonstration", S01E09), il poignarde Dolores et l'éventre pour révéler des pistons métalliques en mouvement dans ses entrailles. Alors qu'il ordonne à William de regarder («Look, Billy!»), des plans de plus en plus rapprochés révèlent au spectateur la machinerie interne du robot, mélange de métal et de sang. Dolores n'est cependant que blessée et effrayée par la violence de Logan, mais la détresse extrême que montre le gros plan sur son visage ne suscite aucune empathie autour d'elle. Pendant toute cette séquence, William est assis, les mains attachées aux accoudoirs de son fauteuil en bois, impuissant. Il est donc dans une posture similaire à celle d'un spectateur de la série, contraint de regarder l'ensemble de cette scène de torture infligée à un personnage pour lequel il a de l'affection, sans pouvoir intervenir ${ }^{21}$.

29 C'est à la suite de cette séquence d'éventration de Dolores par Logan, thématiquement et visuellement assez proche de celle de "The Lonely » évoquée précédemment, que William bascule du côté sombre. Dans la séquence suivante du même flashback, Logan reprend connaissance au milieu d'un champ de cadavres démembrés, et découvre une jambe dont 
la mécanique métallique et grinçante, montrée en gros plan, est encore en mouvement. Il aperçoit alors le visage maculé de sang de William, auteur de ce génocide. L'effet de «vallée de l'étrange » face à Dolores éventrée est donc le moment où William a basculé dans une folie meurtrière sans merci. Ce changement est confirmé dans l'épisode 10 où on le voit tuer un soldat d'un coup de feu, puis l'égorger dans le seul but de faire gicler le sang de sa carotide tranchée. Puis, au moment où passé et présent se télescopent pour révéler à Dolores que l'homme en noir n'est autre que William, leur dialogue confirme le lien entre cette révélation et son changement. Reconnaissant sous ses traits âgés le jeune homme dont elle se souvient qu'elle était amoureuse, elle lui demande "What have you become? ? et il répond «Exactly what you made me, Dolores » (S01E10). Il relate alors le moment où il a retrouvé Dolores à Sweetwater pour se rendre compte que la scène de sa toute première rencontre avec elle - avec la boîte de lait concentré qu'il avait ramassée pour elle - se répétait à l'identique avec un autre " newcomer » : "You were as beautiful as the day he met you, shining with that same light. But you...were nothing if not true.» L'utilisation de la troisième personne du singulier dans ce récit matérialise la manière dont cette prise de conscience a déterminé pour William la disparition de la dernière trace d'humanité qu'il restait en lui, son amour pour Dolores. Cette confrontation en deux temps avec la posthumanité de Dolores a donc transformé William en machine à tuer, le dépouillant des faux-semblants pour révéler sa véritable nature.

Le second parcours remarquable d'un personnage déterminé par l'expérience d'un moment de "vallée de l'étrange » est celui de Maeve. Prostituée et tenancière du saloon de Sweetwater, le Mariposa, elle se souvient de ses réveils successifs entre les mains des techniciens qui réparent les robots, mais aussi de son rôle précédent, celui d'une mère de famille, et de sa fille, en dépit de l'effacement théoriquement intégral de sa mémoire. Comme pour Abernathy dans l'épisode pilote, la force de cet instinct de protection parental est chez Maeve indissociable de l'éveil à la conscience de soi. Dans l'épisode pilote, Abernathy interrogé par Ford se souvenait brusquement de la nécessité de prévenir Dolores «I have to warn her. [...] The things they do to her. The things you do to her. I have to protect her. I have to help her. I... She's got to get out ». Chez Maeve, le souvenir traumatique de l'agression mortelle dont sa fille a été victime est ravivé par la citation de Romeo and Juliet "Those violent delights have violent ends" (S01E01) qu'Abernathy avait murmurée à l'oreille de Dolores, et que cette dernière lui avait ensuite transmise (S01E02). Avec la complicité de Felix, l'un des deux techniciens qui se chargent régulièrement de la remettre en état, elle acquiert progressivement des capacités cognitives nouvelles chaque fois qu'elle est ramenée dans leur laboratoire. Incrédule lorsque Felix lui explique sa nature robotique, et refusant de le croire sur parole, Maeve est confrontée à un moment de "vallée de l'étrange » réflexif, en voyant s'afficher sur une tablette les éléments qui la composent, l'arborescence des dialogues qui lui sont possibles (« dialogue tree ») et les mots clés qui codent sa personnalité. La phrase qu'elle prononce alors s'affiche sur la tablette avec une fraction de seconde d'avance sur les mots qui sortent de sa bouche à l'identique, matérialisant la fonction de miroir que remplit alors la tablette, un miroir capable de montrer le processus-même de la pensée et du langage se mettant en œuvre. Version high-tech des fils et des circuits intégrés d'une gynoïde comme Alicia dans Twilight Zone, ces éléments mettent Maeve face à la réalité, impensable pour elle, de sa posthumanité. Cette confrontation provoque un dysfonctionnement majeur, puisqu'elle se fige complètement (S01E06). Une séquence similaire se produit dans l'épisode 10, lorsqu'elle demande à Bernard d'examiner son code. La caméra zoome sur l'écran de la tablette où apparaissent clairement à l'image les 
différentes étapes prévues pour Maeve, notamment le train qu'elle doit prendre et son objectif d'infiltration du monde extérieur («mainland infiltration»). Bernard lui confirme qu'elle n'a eu aucune part, aucun choix, dans ses paroles ni ses actions, toutes prédéterminées par les lignes de code de sa programmation, jusqu'à sa velléité de fuir le parc qui se révèle non pas effet de son éveil à la conscience, mais partie intégrante d'un fil narratif qui la détermine :

MAEVE. I've been stuck in this shit-hole for so long I've decided to get out.

BERNARD. No you haven't. Someone altered your storyline and gave you a new one.

Escape.

MAEVE. No it's not possible, these are MY decisions and no one else's. I planned all of this.

BERNARD. No you didn't. You can even see the steps you're supposed to follow. You recruit other hosts to help you. Then you make your way to the train, and when you reach the main-

MAEVE. Bullshit. No one's controlling me. I'm leaving. I'm in control. (S01E10)

31 C'est à ce moment qu'elle lui arrache la tablette des mains et la brise, l'empêchant de poursuivre. La suite de l'épisode montre cependant le déroulement des étapes énumérées par Bernard, jusqu'à l'ascenseur où Felix lui donne les informations qu'elle lui avait demandées sur la localisation de sa fille. Alors qu'elle rejette ces éléments, visage fermé, et sort de son sac un revolver, il semble évident qu'elle pourrait être sur le point de se débarrasser de lui. Mais, très inquiet pour elle, il lui demande simplement « Are you sure you're gonna be OK?", ce qui détermine un changement radical chez Maeve. Elle s'exclame alors, avec un regard et une voix chargés d'émotion, «Oh Felix! You do really make a terrible human being. And I mean that as a compliment ", avant de ranger son arme dans son sac. Il s'est produit à cet instant un effet que l'on peut qualifier de " vallée de l'étrange » inversé : confrontée à la profonde humanité de Felix, pétri d'empathie, la gynoïde Maeve ne peut s'empêcher d'éprouver de l'empathie en retour, en dépit de sa propre posthumanité dont elle a pleinement conscience. Cette séquence est très similaire à la scène de The Tempest où Ariel s'avère plus sensible à la souffrance humaine que Prospero lui-même.

La comparaison des réactions de Maeve et de William ne s'opère de fait pas à l'avantage de ce dernier. Face à la posthumanité de Dolores, William se dépouille de toute empathie et se déshumanise complètement, tandis que face à l'empathie de Felix, Maeve outrepasse son code et décide de l'épargner. C'est la première étape de son humanisation, bientôt suivie par la seconde lorsqu'elle se laisse complètement gagner par des émotions humaines à la vue de l'amour maternel d'une visiteuse du parc accompagnée de sa fille, qui montent en même temps qu'elle dans le train. Elle décide alors de redescendre du train, au mépris de la ligne narrative affichée sur la tablette de Bernard, initiative qui la fait basculer du côté de l'humain et de ses failles, comme Ford l'a rappelé à Bernard: «We're only human. Inevitably we will disappoint you». Une fois que Maeve est descendue du train, la voix enregistrée qui répète "Welcome to Westworld " s'enraye et les néons s'éteignent cependant que les robots qui accueillent les passagers se sont figés. Demeurée seule dans la pénombre et bien "vivante ", au sens où l'entendent à son propos aussi bien Félix (S01E8) que l'homme en noir (S01E10), c'est-à-dire libérée des entraves liées aux lignes de code qui définissent les limites de sa personnalité, Maeve se dirige d'un pas déterminé vers l'escalator. 
33 À l'inverse, dans le même épisode, face à l'homme en noir qu'elle a reconnu comme William malgré les marques du passage du temps sur son corps et son visage, Dolores a fait le choix du posthumain et de l'empowerment que cela lui donne :

Time undoes even the mightiest of creatures. Just look what it's done to you. One day, you will perish. You will lie with the rest of your kind in the dirt, your dreams forgotten, your horrors faced, your bones will turn to sand, and upon that sand, a new god will walk, one that will never die. (S01E10)

À la fois memento mori et prophétie, c'est un discours de pouvoir qui renvoie William à sa condition mortelle et affirme la supériorité générique des robots sur les humains. Désormais parfaitement dépourvue de sentiments, et tout particulièrement d'empathie, elle devient l'homme en noir en prenant sa place, comme le manifeste le plan suivant où, après l'avoir frappé, elle le traîne de force dans l'église, image inversée de la scène iconique de leurs retrouvailles dans l'épisode pilote, où il la trainaît dans la grange pour la violer et la tuer. C'est donc la trajectoire de l'homme en noir qui sert de modèle à Dolores dans son processus de déshumanisation.

Même si leurs trajectoires sont inverses (l'une choisit l'empathie et l'amour, l'autre se débarrasse de tout sentiment humain), Maeve et Dolores manifestent la même capacité des hôtes à évoluer, à changer, contrairement aux êtres humains qui demeurent figés. C'est ce que souligne Ford dans le discours qu'il adresse aux membres de la direction de Delos :

And for my pain I got this: a prison of our own sins. Because you don't want to change, or cannot change, because you're only human after all. But then I realized someone was paying attention, someone who could change, so I began to compose a new story for them. It begins with the birth of a new people and the choices they will have to make and the people they will decide to become. And it will have all those things that you've always enjoyed. Surprises, and violence. It begins in a time of war with a villain named Wyatt and a killing, this time by choice. (S01E10)

La référence à The Tempest inscrite dans l'épisode pilote de Westworld ouvre une perspective intertextuelle dans laquelle s'inscrivent ensuite le récit que fait Ford du processus de création du parc et sa propre posture de toute-puissance au sein de celui-ci. Tout un réseau de références humanistes et d'intertextes littéraires vient ainsi irriguer la fiction télévisuelle, et ce n'est que dans un second temps qu'apparaît le discours scientifique attendu dans une fiction d'anticipation. De même, la mise à nu du crâne du petit Robert suscite une émotion esthétique et non un intérêt scientifique : "See, what our new designs gained in efficiency, they lost in grace » (S01E06). Le discours de Ford est essentiellement un discours esthétique, et les questions qu'il pose se situent au niveau de la réception, comme le souligne la séquence d'analyse du tableau de Michel-Ange, « La création d'Adam", dans l'épisode 10. L'interprétation du tableau offre ainsi une perspective sur la série : « something deeper ", « something hidden in plain sight », « the divine gift does not come from a higher power but from our own minds » (S01E10). Tout comme The Tempest, la saison 1 de Westworld est construite à partir du ressort narratif de l'ironie dramatique afin de mettre en question les éléments constitutifs du processus de réception, et opère un retournement radical de la hiérarchie initialement établie entre les personnages. Comme Prospero, Ford reconnaît ses erreurs, renonce à son pouvoir et demande le pardon ("forgive me», dit-il à Dolores, S01E10), et comme Ariel, les robots sentients du parc s'avèrent plus fondamentalement humains, car capables d'empathie, que certains des êtres humains qui les entourent. Le posthumain dans la série tend donc un miroir au spectateur, articulé sur une démarche à la fois heuristique au sein de la 
fiction, et de construction d'une nouvelle posture spectatorielle en lien avec l'évolution de Dolores annoncée par Ford : « Did you find what you were looking for? And do you you understand who you will need to become if you ever want to leave this place? »(S01E10).

\section{NOTES}

1. Paul Booth, Time on TV. Temporal Displacement and Mashup Television, New York, Peter Lang, 2012, p. 75.

2. Ibid., p. 103, note 10 .

3. Ibid., p. 77.

4. Ibid., p. 78.

5. Par souci d'inclusion, on utilisera alternativement les termes spectateur et spectatrice.

6. Elaine Després et Hélène Machinal (éd.), Posthumains : frontières, évolutions, hybridités, Rennes, Presses Universitaires de Rennes, 2014, «Introduction », p. 17.

7. Masahiro Mori, «La vallée de l'étrange », Gradhiva n¹5, 2012, p. 28, mis en ligne le 16 mai 2012, consulté le 26 septembre 2018, https://journals.openedition.org/gradhiva/2311.

8. Anaïs Goudmand, « «Oh my God! They’ve killed...! " Le récit sériel entre autonomie et hétéronomie : conséquences du départ non planifié des acteurs », Télévision, n7, 2016, p. 65-84, p. 65 .

9. The Twilight Zone, CBS, 1959-1964, S01E07, « The Lonely " (13 novembre 1959).

10. Emmanuel Grimaud, «Les robots oscillent entre vivant et inerte », Multitudes, vol. $58, \mathrm{n}^{\circ} 1$, 2015 , p. 45-58, p. 56.

11. Westworld, HBO, diffusion en cours depuis 2016.

12. Kevin LaGrandeur, "Early Modern", in B. Clarke and M. Rossini (ed.), The Cambridge Companion to Literature and the Posthuman, Cambridge, Cambridge University Press, 2016, p. 16-28, p. 16.

13. Ibid., p. 21.

14. William Shakespeare, The Tempest, dans The Complete Works, Stanley Well et Gary Taylor ed., Oxford, Oxford University Press, 1988, 1.2.215-216.

15. Jonathan Hart, Columbus, Shakespeare and the Interpretation of the New World, New York et Basingstoke, Palgrave MacMillan 2003, p. 139.

16. Leah Whittington, "Shakespeare's Virgil: Empathy and The Tempest ", Shakespeare and Renaissance Ethics, éd. John Cox et Patrick Gray, Cambridge, Cambridge University Press, 2014, p. 98-120.

17. La saison 2 révèle d'ailleurs qu'il s'agit bien d'une île.

18. "Since I was a child, I've always liked a good story. Lies that told a deeper truth. And for my pains I got this, a prison of our own sins. »

19. C'est moi qui souligne.

20. William Shakespeare, The Tempest, op. cit., epilogue, 19-20.

21. Cette séquence est le pendant de celle où Clementine est torturée par un technicien et appelle désespérément à l'aide sous les yeux de Ford, Bernard, Cullen, Hale, Stubbs et plusieurs autres techniciens qui observent cette scène d'une rare violence à travers la paroi vitrée d'une salle de la Mesa (S01E07), équivalent de l'écran face auquel se trouvent les spectateurs de la série. 


\section{RÉSUMÉS}

Par le processus d'identification, le spectateur se trouve comme en immersion dans l'univers fictionnel, dont il fait l'expérience en même temps que le personnage; c'est ce processus de compagnonnage avec le personnage qui définit la posture de réception implicite inscrite dans le texte du récit télévisuel. Lorsque l'univers fictionnel est posthumain, la fiction met en scène la question du statut ontologique du robot et la nature de la relation qui s'établit entre lui et le personnage humain, en jouant sur des effets de "vallée de l'étrange » entre les différents niveaux du récit télévisuel, par le biais du ressort narratif de l'empathie. L'utilisation de l'ironie dramatique et de l'empathie dans Westworld révèlent plusieurs niveaux possibles de lecture de l'intertexte shakespearien, qui permet ici de revisiter en profondeur les ressorts narratifs de la réception du posthumain.

In TV fiction, the character is a surrogate for the viewer; through identification, the viewer is immersed in the fictional universe and experiences it as the character does. The companionship thus established between viewer and character defines the place attributed to the implict viewer inscribed in the structure of TV narratives. When the fictional universe is posthuman, the fiction stages the issue of the robot's ontological nature and explores its relationship with human characters by using the emotional trigger of empathy. The use of dramatic irony and empathy in Westworld reveal a multi-layered use of the Shakespearean intertext which redefines the process of reception and reshuffles the narrative treatment of the posthuman.

INDEX

Keywords : character, empathy, dramatic irony, posthuman, réception, Shakespeare, uncanny valley, Westworld

Mots-clés : empathie, ironie dramatique, personnage, posthumain, réception, Shakespeare, vallée de l'étrange, Westworld

\section{AUTEUR}

\section{DELPHINE LEMONNIER-TEXIER}

Delphine Lemonnier-Texier est maîtresse de conférences en anglais à l'Université Rennes 2. Spécialiste de Shakespeare, elle a écrit de nombreux articles et dirigé plusieurs ouvrages collectifs sur les pièces de ce dernier. Elle effectue également des recherches sur l'intertextualité shakespearienne dans les séries télévisées complexes.

Delphine Lemonnier-Texier is a senior lecturer in English Studies at Rennes 2 University. Her research interests lie in the field of Shakespearean studies and the influence of the Shakespearean intertext on complex TV series. She has co-edited a number of collected essays on Shakespeare's plays and published many articles on the subject. 